\title{
Exposure Order Effects and Advertising Competition
}

\author{
Oksana Loginova \\ University of Missouri-Columbia \\ Department of Economics \\ 118 Professional Bldg \\ Columbia, MO 65211 \\ loginovao@missouri.edu
}

May 10, 2008

\begin{abstract}
This paper applies the theories of exposure order effects, developed in the psychology literature, to an industrial organization model to explore their role in advertising competition. There are two firms and infinitely many identical consumers. The firms produce a homogeneous product and distribute their brands through a common retailer. Consumers randomly arrive at the retailer and buy their most preferred brands. The order in which a consumer sees the advertising messages affects his brand preferences. Under the primacy effect the consumer prefers the brand he first saw advertised, under the recency - the last encountered brand. The equilibrium of the advertising game is characterized separately under the primacy and the recency effects. In the first setting all consumers are initially unaware of the product existence. The equilibrium advertising intensities, remarkably, do not depend on the type of exposure order effect. In the other two settings some consumers have already formed their brand preferences. The primacy and the recency effects give rise to different equilibrium outcomes.
\end{abstract}

JEL Classifications: C73, D11, D43, L13, M37.

Keywords: advertising order effects, primacy, recency. 


\section{Introduction}

Traditionally, advertising has been studied by two very different subfields of social science, economics and psychology. ${ }^{1}$ The economic literature on advertising has been concerned with such important questions as the informational content of ads, the socially optimal level of advertising, and firm rivalry. The psychology literature, among other things, has explored the connection between information processing and advertising effectiveness. The goal of this paper is to develop a model that incorporates economics and psychology to examine how information processing biases affect advertising competition between firms.

Past research in psychology has shown that the order in which consumers are exposed to brands influence consumer preferences. Two advertising order effects have received attention. The primacy effect is characterized by greater persuasion consequence of the first advertising message. On the other hand, the recency effect occurs when the last encountered brand is preferred. Whether exposure order results in the primacy effect or in the recency, depends on different factors, such as overall involvement, motivation, attitude strength, and the time between information exposure and preference construction.

Lana (1963) showed that subjects greatly interested in the topic exhibited the primacy effect, whereas subjects with minimal interest exhibited the recency effect. The primacy effect occurs when participants are motivated to elaborate the initial message and show critical thinking toward later information, whereas the recency effect occurs when motivation is low (Haugtvedt and Wegner 1994). Brunel and Nelson (2003) examined how advertising order effects connect to gender differences. Their results suggested that under conditions of low involvement, females exhibited the primacy effect, males - the recency effect. Under conditions of higher involvement, females continued to exhibit the primacy effect, the recency effect with male disappeared when the advertisement matched their values. Neidrich and Swain (2007) found the primacy effect when the delay between attribute encoding and preference construction is long (a few days). On the other hand, the recency effect is more likely to occur when the delay is short (a few minutes).

\footnotetext{
${ }^{1}$ Of course, advertising is one of the primary subjects studied in the field of marketing. Most papers in marketing concerning advertising, however, can be classified as having either economic or psychologic foundations.
} 
The model presented here applies the primacy and the recency effects to advertising competition. The agents are two firms and infinitely many identical consumers of total mass one. The firms produce a homogeneous product; firm $A$ produces brand $A$ and firm $B$ produces brand $B$. The firms distribute their brands through a common retailer. Each consumer randomly (according to a Poisson process) arrives at the retailer and buys his most preferred brand. Consumers learn about the product existence and form their brand preferences through the firms' advertising. The order in which a consumer sees the advertising messages affects his brand preferences. Under the primacy effect the consumer purchases the brand he first saw advertised, whereas under the recency effect - whichever brand he most recently saw advertised.

The firms compete in advertising intensities - the rate parameters of Poisson processes that advertising messages follow. The equilibrium of the advertising game is characterized separately under the primacy and the recency effects. Three setting are considered. In Setting 1 (New Market) consumers initially are unaware of the product existence. In this setup, the equilibrium advertising intensities are symmetric and, remarkably, do not depend on the type of exposure order effect.

In Setting 2 (Growing Market) it is assumed that at the beginning of the game a number of consumers believe brand $A$ is better, the equal number prefer brand $B$, and the rest are unaware of the product existence. In this setup the two exposure order effects give rise to different equilibrium outcomes. Results show that the firms choose higher advertising intensities under the recency effect than under the primacy. In Setting 3 firm $A$ has advantage over firm $B$. Specifically, at the beginning of the game a number of consumers prefer brand $A$ and the rest are unaware of the product existence. Under the primacy effect the equilibrium advertising intensities are symmetric, whereas under the recency effect firm $B$ chooses higher advertising intensity than firm $A$. Various welfare implications are investigated.

This paper contributes to the small but growing body of economic literature on advertising grounded in psychological research. Krähmer (2004) developed a monopolistic model in which the buyer may not recall correctly his past experience with the product. Advertising 
can activate memory and help consumers to recollect their past experiences, or distort actual consumption experiences. Shapiro (2006) explored two alternative advertising mechanisms that yield very different predictions. In the first, advertising converts memories of bad experiences into memories of good ones. In the second, advertising makes favorable experiences more likely to be remembered. Brekke and Rege (2007) captured availability heuristic phenomenon, according to which consumers cannot distinguish between the recommendations or real people and fictitious characters in advertisements. Their analysis showed that even if a person knows that his observations of others may be distorted by advertising, it is still rational for him to choose whichever product he has observed most often.

In the next section, the formal model is presented. The three settings are analyzed in sections III through VI. Concluding remarks appear in Section VII. All proofs are relegated to the Appendix. 


\section{The Model}

\section{Firms and Consumers}

Two firms, $A$ and $B$, produce a homogeneous product using zero marginal cost technology. The firms distribute their brands through a common retailer. The demand side consists of infinitely many identical consumers of total mass 1 . Consumers are risk-neutral, possess continuous-time discount rate $r>0$, and have a sequence of unit demands for the product. In particular, a consumer receives a gross payoff of 1 whenever he arrives at the retailer and buys his most preferred brand. The number of times the consumer visits the retailer follows a homogenous Poisson process with rate parameter $\sigma>0$. That is, in a small time interval $\Delta t$ the consumer visits the retailer with infinitesimal probability $\sigma \Delta t+o(\Delta t){ }^{2}$

Consumers learn about the product existence and form their brand preferences through advertising. The number of ads describing brand $A$ a consumer receives is a homogeneous Poisson process with rate parameter $\alpha_{A}$, the number of ads describing brand $B$ ads he receives follows a Poisson process with rate parameter $\alpha_{B}$. It is assumed that consumers receive advertising messages independently from each other (they watch/ listen to different TV/ radio programs at different times).

Advertising intensities, $\alpha_{A}$ and $\alpha_{B}$, are chosen by the firms. The discounted cost needed to achieve intensity $\alpha \geq 0$ is $c(\alpha) / r$. Function $c(\cdot)$ is convex and increasing, $c^{\prime}>0$ and $c^{\prime \prime}>0$. The standard boundary assumptions, $c(0)=c^{\prime}(0)=0$ and $\lim _{\alpha \rightarrow \infty} c^{\prime}(\alpha)=\infty$, guarantee the existence of an interior solution.

\section{Advertising Order Effects}

The order in which a consumer sees the advertising messages affects his brand preferences. Two main effects are considered: primacy and recency. The primacy effect is characterized by greater persuasion consequence of the first advertisement. In the context of the present

\footnotetext{
${ }^{2}$ Formally, let $N_{t}$ denote the number of times a consumer visits the retailer by time $t . N_{t}$ is a Poisson process with rate parameter $\sigma>0$ if and only if (i) the number of visits during one time interval is independent of the number of visits during a different non-overlapping time interval; (ii) in a small time interval $\Delta t$ the consumer visits the retailer with probability $\operatorname{Pr}\left\{N_{t+\Delta t}=N_{t}+1\right\}=\sigma \Delta t+o(\Delta t)$, and he visits the retailer more than one time with probability $o(\Delta t)$. On Poisson processes see, for example, Doob (1953).
} 
model, the primacy effect implies that the consumer purchases whichever brand he first saw advertised. The recency effect, on the other hand, occurs when the last encountered brand is preferred. That is, the consumer buys whichever brand he most recently saw advertised.

Let $x_{A}(t)$ denote the number of consumers that at time $t$ believe brand $A$ is better. Similarly, $x_{B}(t)$ is the number of consumers that prefer brand $B$. The rest of the consumers, $x_{\varnothing}(t)=1-x_{A}(t)-x_{B}(t)$, are unaware of the product existence. These consumers will be referred to as "ignorant" consumers, for the lack of a better term. How do $x_{A}(t), x_{B}(t)$, and $x_{\varnothing}(t)$ evolve under the primacy and the recency effects given advertising intensities $\alpha_{A}$ and $\alpha_{B} ?$

Consider the primacy effect. The probability that a consumer sees firm $A$ 's advertisement in a small time interval $\Delta t$ is $\alpha_{A} \Delta t+o(\Delta t)$. Hence, the number of ignorant consumers who see firm $A$ 's ad is

$$
\alpha_{A} x_{\varnothing}(t) \Delta t+o(\Delta t)
$$

Similarly, the number of ignorant consumers who see firm $B$ 's ad is

$$
\alpha_{B} x_{\varnothing}(t) \Delta t+o(\Delta t)
$$

Therefore, $x_{A}(t)$ increases at instantaneous rate $\alpha_{A} x_{\varnothing}(t), x_{B}(t)$ increases at rate $\alpha_{B} x_{\varnothing}(t)$, and the number of ignorant consumers decreases at rate $\left(\alpha_{A}+\alpha_{B}\right) x_{\varnothing}(t)$. Algebraically,

$$
\left\{\begin{array}{l}
\frac{\mathrm{d}}{\mathrm{d} t} x_{\varnothing}(t)=-\left(\alpha_{A}+\alpha_{B}\right) x_{\varnothing}(t) \\
\frac{\mathrm{d}}{\mathrm{d} t} x_{A}(t)=\alpha_{A} x_{\varnothing}(t) \\
\frac{\mathrm{d}}{\mathrm{d} t} x_{B}(t)=\alpha_{B} x_{\varnothing}(t)
\end{array}\right.
$$

The recency effect differs from the primacy effect in that consumers switch from one brand to the other when they see an advertisement for the latter. Thus, the number of consumers who switch from brand $A$ to brand $B$ is

$$
\alpha_{B} x_{A}(t) \Delta t+o(\Delta t)
$$


Similarly, the number of consumers who switch from brand $B$ to brand $A$ is

$$
\alpha_{A} x_{B}(t) \Delta t+o(\Delta t)
$$

Therefore, $x_{A}(t)$ increases at rate $\alpha_{A}\left(x_{\varnothing}(t)+x_{B}(t)\right)$ and decreases at rate $\alpha_{B} x_{A}(t), x_{B}(t)$ increases at rate $\alpha_{B}\left(x_{\varnothing}(t)+x_{A}(t)\right)$ and decreases at rate $\alpha_{A} x_{B}(t)$. Algebraically,

$$
\left\{\begin{array}{l}
\frac{\mathrm{d}}{\mathrm{d} t} x_{\varnothing}(t)=-\left(\alpha_{A}+\alpha_{B}\right) x_{\varnothing}(t), \\
\frac{\mathrm{d}}{\mathrm{d} t} x_{A}(t)=\alpha_{A}\left(x_{\varnothing}(t)+x_{B}(t)\right)-\alpha_{B} x_{A}(t), \\
\frac{\mathrm{d}}{\mathrm{d} t} x_{B}(t)=\alpha_{B}\left(x_{\varnothing}(t)+x_{A}(t)\right)-\alpha_{A} x_{B}(t) .
\end{array}\right.
$$

The transition matrices (infinitesimal generators) for the primacy and the recency effects are, respectively,

$$
P=\left(\begin{array}{ccc}
-\left(\alpha_{A}+\alpha_{B}\right) & 0 & 0 \\
\alpha_{A} & 0 & 0 \\
\alpha_{B} & 0 & 0
\end{array}\right)
$$

and

$$
R=\left(\begin{array}{ccc}
-\left(\alpha_{A}+\alpha_{B}\right) & 0 & 0 \\
\alpha_{A} & -\alpha_{B} & \alpha_{A} \\
\alpha_{B} & \alpha_{B} & -\alpha_{A}
\end{array}\right) .
$$

Note that the column sums of $P$ and $R$ are equal to zero, the nondiagonal entries are nonnegative, and the diagonal entries are nonpositive. Let

$$
\bar{x}(t)=\left(\begin{array}{c}
x_{\varnothing}(t) \\
x_{A}(t) \\
x_{B}(t)
\end{array}\right) .
$$

Then the systems of differential equations (1) and (2) can be rewritten in a matrix form,

$$
\frac{d}{d t} \bar{x}(t)=P \bar{x}(t)
$$


and

$$
\frac{d}{d t} \bar{x}(t)=R \bar{x}(t)
$$

The solutions are given by

$$
\bar{x}(t)=e^{t P} \bar{x}(0)
$$

and

$$
\bar{x}(t)=e^{t R} \bar{x}(0)
$$

where $\bar{x}(0)$ is the vector of initial conditions. ${ }^{3}$

\section{Profit Functions of the Firms}

The diagonalization ${ }^{4}$ is used to compute the exponentials $e^{t P}$ and $e^{t R}$,

$$
e^{t P}=\left(\begin{array}{ccc}
e^{-\left(\alpha_{A}+\alpha_{B}\right) t} & 0 & 0 \\
\frac{\alpha_{A}-\alpha_{A} e^{-\left(\alpha_{A}+\alpha_{B}\right) t}}{\alpha_{A}+\alpha_{B}} & 1 & 0 \\
\frac{\alpha_{B}-\alpha_{B} e^{-\left(\alpha_{A}+\alpha_{B}\right) t}}{\alpha_{A}+\alpha_{B}} & 0 & 1
\end{array}\right)
$$

and

$$
e^{t R}=\left(\begin{array}{ccc}
e^{-\left(\alpha_{A}+\alpha_{B}\right) t} & 0 & 0 \\
\frac{\alpha_{A}-\alpha_{A} e^{-\left(\alpha_{A}+\alpha_{B}\right) t}}{\alpha_{A}+\alpha_{B}} & \frac{\alpha_{A}+\alpha_{B} e^{-\left(\alpha_{A}+\alpha_{B}\right) t}}{\alpha_{A}+\alpha_{B}} & \frac{\alpha_{A}-\alpha_{A} e^{-\left(\alpha_{A}+\alpha_{B}\right) t}}{\alpha_{A}+\alpha_{B}} \\
\frac{\alpha_{B}-\alpha_{B} e^{-\left(\alpha_{A}+\alpha_{B}\right) t}}{\alpha_{A}+\alpha_{B}} & \frac{\alpha_{B}-\alpha_{B} e^{-\left(\alpha_{A}+\alpha_{B}\right) t}}{\alpha_{A}+\alpha_{B}} & \frac{\alpha_{B}+\alpha_{A} e^{-\left(\alpha_{A}+\alpha_{B}\right) t}}{\alpha_{A}+\alpha_{B}}
\end{array}\right) .
$$

Straightforward but tedious calculations are relegated to the Appendix. Substituting the above expressions into (3) and (4) allows to compute $\bar{x}(t)$ under the primacy and the recency effects, hence the number of consumers that prefer brand $A, x_{A}(t)$, and the number of consumers that

${ }^{3}$ The exponential $e^{t A}$ is defined as

$$
e^{t A}=\sum_{n=0}^{\infty} \frac{(t A)^{n}}{n !}
$$

${ }^{4}$ The first step is to diagonalize the matrix, $A=Q D Q^{-1}$, where $D=\operatorname{diag}\left(d_{1}, d_{2}, \ldots\right)$. Once $A$ is diagonalized it is easy to compute exponential $e^{t A}$,

$$
e^{t A}=\sum_{n=0}^{\infty} \frac{Q(t D)^{n} Q^{-1}}{n !}=Q \operatorname{diag}\left(e^{d_{1} t}, e^{d_{2} t}, \ldots\right) Q^{-1}
$$


believe brand $B$ is better, $x_{B}(t)$.

There is no price competition between the firms, as, by assumption, each consumer buys whichever brand he believes is better. The firms set their prices equal to 1 , consumer valuation for the product. Hence, the profit functions of the firms are

$$
\Pi_{A}\left(\alpha_{A}, \alpha_{B}\right)=\int_{0}^{\infty} e^{-r t} x_{A}(t) \sigma d t-\frac{c\left(\alpha_{A}\right)}{r}
$$

and

$$
\Pi_{B}\left(\alpha_{A}, \alpha_{B}\right)=\int_{0}^{\infty} e^{-r t} x_{B}(t) \sigma d t-\frac{c\left(\alpha_{B}\right)}{r} .
$$

\section{Three Different Settings}

The firms choose their advertising intensities $\alpha_{A}$ and $\alpha_{B}$ simultaneously at the beginning of the game. The equilibrium concept employed is Nash equilibrium. Three different settings are considered. In Setting 1 it is assumed that at time $t=0$ consumers are unaware of the product existence,

$$
\bar{x}(0)=\left(\begin{array}{l}
1 \\
0 \\
0
\end{array}\right) .
$$

In Setting 2, $\gamma$ consumers are ignorant, $(1-\gamma) / 2$ believe brand $A$ is better, and $(1-\gamma) / 2$ prefer brand $B$. That is,

$$
\bar{x}(0)=\left(\begin{array}{c}
\gamma \\
\frac{1}{2}(1-\gamma) \\
\frac{1}{2}(1-\gamma)
\end{array}\right) .
$$

In Setting 3 firm $A$ has advantage over firm $B$. In particular, it is assumed that at the beginning of the game $\gamma$ consumers are unaware of the product existence, the rest believe brand $A$ is better,

$$
\bar{x}(0)=\left(\begin{array}{c}
\gamma \\
1-\gamma \\
0
\end{array}\right) \text {. }
$$


In the next three sections each setting is analyzed separately under the primacy and the recency effects.

\section{Setting 1 (New Market)}

Consider the setting in which initially consumers are unaware of the product existence. Under the primacy effect,

$$
\bar{x}(t)=\left(\begin{array}{ccc}
e^{-\left(\alpha_{A}+\alpha_{B}\right) t} & 0 & 0 \\
\frac{\alpha_{A}-\alpha_{A} e^{-\left(\alpha_{A}+\alpha_{B}\right) t}}{\alpha_{A}+\alpha_{B}} & 1 & 0 \\
\frac{\alpha_{B}-\alpha_{B} e^{-\left(\alpha_{A}+\alpha_{B}\right) t}}{\alpha_{A}+\alpha_{B}} & 0 & 1
\end{array}\right)\left(\begin{array}{l}
1 \\
0 \\
0
\end{array}\right)=\left(\begin{array}{c}
e^{-\left(\alpha_{A}+\alpha_{B}\right) t} \\
\frac{\alpha_{A}-\alpha_{A} e^{-\left(\alpha_{A}+\alpha_{B}\right) t}}{\alpha_{A}+\alpha_{B}} \\
\frac{\alpha_{B}-\alpha_{B} e^{-\left(\alpha_{A}+\alpha_{B}\right) t}}{\alpha_{A}+\alpha_{B}}
\end{array}\right) .
$$

Hence,

$$
x_{A}(t)=\frac{\alpha_{A}-\alpha_{A} e^{-\left(\alpha_{A}+\alpha_{B}\right) t}}{\alpha_{A}+\alpha_{B}}
$$

and

$$
x_{B}(t)=\frac{\alpha_{B}-\alpha_{B} e^{-\left(\alpha_{A}+\alpha_{B}\right) t}}{\alpha_{A}+\alpha_{B}} .
$$

Observe that the first column of matrix (5) coincides with the first column of matrix (6). Hence, functions $x_{A}(t)$ and $x_{B}(t)$ are the same under the recency effect as under the primacy. This apparently surprising result can be easily explained. Indeed, the number of consumers who switch from brand $B$ to brand $A$ in a small time interval $\Delta t$ is

$$
\alpha_{A} x_{B}(t) \Delta t+o(\Delta t) \text {. }
$$

Similarly,

$$
\alpha_{B} x_{A}(t) \Delta t+o(\Delta t)
$$

consumers switch in the opposite direction - from brand $A$ to brand $B$. It follows from (7) and (8) that for any given $\alpha_{A}$ and $\alpha_{B}$

$$
\alpha_{A} x_{B}(t)=\alpha_{B} x_{A}(t)
$$


holds for all values of $t$. Therefore, consumer switching does not affect $x_{A}(t)$ and $x_{B}(t)$ obtained under the primacy effect.

Substituting (7) and (8) into the firms' profit functions and computing the integrals yield

$$
\Pi_{A}\left(\alpha_{A}, \alpha_{B}\right)=\frac{\sigma \alpha_{A}}{r\left(\alpha_{A}+\alpha_{B}+r\right)}-\frac{c\left(\alpha_{A}\right)}{r}
$$

and

$$
\Pi_{B}\left(\alpha_{A}, \alpha_{B}\right)=\frac{\sigma \alpha_{B}}{r\left(\alpha_{A}+\alpha_{B}+r\right)}-\frac{c\left(\alpha_{B}\right)}{r} .
$$

Let $\alpha_{A}^{*}$ and $\alpha_{B}^{*}$ denote the advertising intensities chosen by the firms in equilibrium.

Proposition 1 (Equilibrium in Setting 1). Under both the primacy and the recency effects $\alpha_{A}^{*}=\alpha_{B}^{*}=\alpha^{*}$, where $\alpha^{*}$ is implicitly defined by

$$
\frac{\sigma\left(\alpha^{*}+r\right)}{\left(2 \alpha^{*}+r\right)^{2}}=c^{\prime}\left(\alpha^{*}\right)
$$

How do the equilibrium levels of advertising intensities compare with the social optimum? The social welfare is given by

$$
W\left(\alpha_{A}, \alpha_{B}\right) \equiv \int_{0}^{\infty} e^{-r t}\left(x_{A}(t)+x_{B}(t)\right) \sigma d t-\frac{1}{r}\left(c\left(\alpha_{A}\right)+c\left(\alpha_{B}\right)\right) .
$$

In the present model it coincides with the sum of the firms' profits. Hence, in Setting 1 the social welfare equals

$$
W\left(\alpha_{A}, \alpha_{B}\right)=\frac{\sigma\left(\alpha_{A}+\alpha_{B}\right)}{r\left(\alpha_{A}+\alpha_{B}+r\right)}-\frac{1}{r}\left(c\left(\alpha_{A}\right)+c\left(\alpha_{B}\right)\right) .
$$

Let $\alpha_{A}^{\dagger}$ and $\alpha_{B}^{\dagger}$ denote the socially optimal advertising intensities.

The next proposition shows that the firms over-advertise in equilibrium. Intuitively, each firm cares only about the number of consumers that prefer its own brand. The social planner, on the other hand, aims to increase the number of consumers that learn about the product existence through the firms' advertising messages, $x_{A}(t)+x_{B}(t)$. 
Proposition 2 (Social Optimum in Setting 1). The socially optimal levels of advertising intensities are $\alpha_{A}^{\dagger}=\alpha_{B}^{\dagger}=\alpha^{\dagger}$, where $\alpha^{\dagger}$ is implicitly defined by

$$
\frac{\sigma r}{\left(2 \alpha^{\dagger}+r\right)^{2}}=c^{\prime}\left(\alpha^{\dagger}\right)
$$

Advertising is socially excessive, $\alpha^{*}>\alpha^{\dagger}$.

In the simple setting with $x_{\varnothing}(0)=1$, the equilibrium outcome is the same under the primacy and the recency effects. However, if at $t=0$ a number of consumers have already formed their preferences about the two brands, as in Setting 2, or if one of the firms has competitive advantage, as in Setting 3 , the two advertising order effects have different strategic implications.

\section{Setting 2 (Growing Market)}

Consider the setting in which at the beginning of the game $(1-\gamma) / 2$ consumers believe brand $A$ is better, $(1-\gamma) / 2$ consumers prefer brand $B$, and the rest are ignorant consumers. Under the primacy effect,

$$
\bar{x}(t)=\left(\begin{array}{ccc}
e^{-\left(\alpha_{A}+\alpha_{B}\right) t} & 0 & 0 \\
\frac{\alpha_{A}-\alpha_{A} e^{-\left(\alpha_{A}+\alpha_{B}\right) t}}{\alpha_{A}+\alpha_{B}} & 1 & 0 \\
\frac{\alpha_{B}-\alpha_{B} e^{-\left(\alpha_{A}+\alpha_{B}\right) t}}{\alpha_{A}+\alpha_{B}} & 0 & 1
\end{array}\right)\left(\begin{array}{c}
\gamma \\
\frac{1}{2}(1-\gamma) \\
\frac{1}{2}(1-\gamma)
\end{array}\right) .
$$

Hence,

$$
x_{A}^{P}(t)=\gamma \frac{\alpha_{A}-\alpha_{A} e^{-\left(\alpha_{A}+\alpha_{B}\right) t}}{\alpha_{A}+\alpha_{B}}+\frac{1}{2}(1-\gamma)
$$

and

$$
x_{B}^{P}(t)=\gamma \frac{\alpha_{B}-\alpha_{B} e^{-\left(\alpha_{A}+\alpha_{B}\right) t}}{\alpha_{A}+\alpha_{B}}+\frac{1}{2}(1-\gamma)
$$


(Superscript "P" stands for primacy.) Substituting (9) and (10) into the firms' profit functions and computing the integrals yield

$$
\Pi_{A}^{P}\left(\alpha_{A}, \alpha_{B}\right)=\gamma \frac{\sigma \alpha_{A}}{r\left(\alpha_{A}+\alpha_{B}+r\right)}+\frac{1}{2}(1-\gamma) \frac{\sigma}{r}-\frac{c\left(\alpha_{A}\right)}{r}
$$

and

$$
\Pi_{B}^{P}\left(\alpha_{A}, \alpha_{B}\right)=\gamma \frac{\sigma \alpha_{B}}{r\left(\alpha_{A}+\alpha_{B}+r\right)}+\frac{1}{2}(1-\gamma) \frac{\sigma}{r}-\frac{c\left(\alpha_{B}\right)}{r} .
$$

These formulas deserve some discussion. At the beginning of the game, $(1-\gamma) / 2$ consumers prefer brand $A$ and - under the primacy effect - will never switch to brand $B$ (they are "locked up" by firm $A$ ). Firm $A$ earns

$$
\frac{1}{2}(1-\gamma) \frac{\sigma}{r}
$$

from these consumers. Similarly, $(1-\gamma) / 2$ consumers are locked up by firm $B$. Firm $B$ earns

$$
\frac{1}{2}(1-\gamma) \frac{\sigma}{r}
$$

from these consumers. Finally, $\gamma$ consumers are unaware of the product existence. If an ignorant consumer first sees an ad describing brand $A$, he becomes firm $A$ 's customer. If the consumer first sees firm $B$ 's ad, he becomes firm $B$ 's customer. Firms $A$ and $B$ earn, respectively,

$$
\gamma \frac{\sigma \alpha_{A}}{r\left(\alpha_{A}+\alpha_{B}+r\right)}
$$

and

$$
\gamma \frac{\sigma \alpha_{B}}{r\left(\alpha_{A}+\alpha_{B}+r\right)}
$$

from $\gamma$ initially ignorant consumers.

In contrast to Setting 1, the recency effect in Setting 2 gives rise to a different vector $\bar{x}(t){ }^{5}$

\footnotetext{
${ }^{5}$ Recall that in Setting $1, \alpha_{A} x_{B}(t)=\alpha_{B} x_{A}(t)$ holds for any values of $\alpha_{A}$ and $\alpha_{B}$. Here, $\alpha_{A} x_{B}^{P}(t)=\alpha_{B} x_{A}^{P}(t)$ holds only for $\alpha_{A}=\alpha_{B}$.
} 
The profits of the firms are given by

$$
\Pi_{A}^{R}\left(\alpha_{A}, \alpha_{B}\right)=\gamma \frac{\sigma \alpha_{A}}{r\left(\alpha_{A}+\alpha_{B}+r\right)}+\frac{1}{2}(1-\gamma) \frac{\sigma}{r}+\frac{1}{2}(1-\gamma) \frac{\sigma\left(\alpha_{A}-\alpha_{B}\right)}{r\left(\alpha_{A}+\alpha_{B}+r\right)}-\frac{c\left(\alpha_{A}\right)}{r}
$$

and

$$
\Pi_{B}^{R}\left(\alpha_{A}, \alpha_{B}\right)=\gamma \frac{\sigma \alpha_{B}}{r\left(\alpha_{A}+\alpha_{B}+r\right)}+\frac{1}{2}(1-\gamma) \frac{\sigma}{r}+\frac{1}{2}(1-\gamma) \frac{\sigma\left(\alpha_{B}-\alpha_{A}\right)}{r\left(\alpha_{A}+\alpha_{B}+r\right)}-\frac{c\left(\alpha_{B}\right)}{r} .
$$

(Superscript "R" stands for recency.) It follows that firm $A$ 's profit function under the recency effect (13) differs from that under the primacy effect (11) by

$$
\frac{1}{2}(1-\gamma) \frac{\sigma\left(\alpha_{A}-\alpha_{B}\right)}{r\left(\alpha_{A}+\alpha_{B}+r\right)}
$$

This term reflects consumer switching between the brands. It enters with negative sign firm $B$ 's profit function (14).

Let $\alpha_{A}^{P *}$ and $\alpha_{B}^{P *}$ denote the advertising intensities chosen by the firms in equilibrium under the primacy effect. Similarly, let $\alpha_{A}^{R *}$ and $\alpha_{B}^{R *}$ denote the equilibrium intensities chosen under the recency effect. Proposition 3 shows that the equilibrium advertising intensities are higher under the recency effect. This result is driven by (15), which creates extra incentives for the firms to advertise.

Proposition 3 (Equilibrium in Setting 2).

(i) Under the primacy effect $\alpha_{A}^{P *}=\alpha_{B}^{P *}=\alpha^{P *}$, where $\alpha^{P *}$ is implicitly defined by

$$
\gamma \frac{\sigma\left(\alpha^{P *}+r\right)}{\left(2 \alpha^{P *}+r\right)^{2}}=c^{\prime}\left(\alpha^{P *}\right)
$$

(ii) Under the recency effect $\alpha_{A}^{R *}=\alpha_{B}^{R *}=\alpha^{R *}$, where $\alpha^{R *}$ is implicitly defined by

$$
\gamma \frac{\sigma\left(\alpha^{R *}+r\right)}{\left(2 \alpha^{R *}+r\right)^{2}}+\frac{1}{2}(1-\gamma) \frac{\sigma}{2 \alpha^{R *}+r}=c^{\prime}\left(\alpha^{R *}\right) .
$$

(iii) There is more advertising under the recency effect, $\alpha^{R *}>\alpha^{P *}$. 
The social welfare is the same under the primacy and the recency effects,

$$
W\left(\alpha_{A}, \alpha_{B}\right)=\gamma \frac{\sigma\left(\alpha_{A}+\alpha_{B}\right)}{r\left(\alpha_{A}+\alpha_{B}+r\right)}+(1-\gamma) \frac{\sigma}{r}-\frac{1}{r}\left(c\left(\alpha_{A}\right)+c\left(\alpha_{B}\right)\right) .
$$

Indeed, it does not matter for the social planner whether a consumer switches from one brand to the other after he observes an advertising message for the latter (the recency effect), or not (the primacy effect). The next proposition shows that the firms over-advertise under the primacy effect, and, hence, under the recency effect.

Proposition 4 (Social Optimum in Setting 2). The socially optimal levels of advertising intensities are $\alpha_{A}^{\dagger}=\alpha_{B}^{\dagger}=\alpha^{\dagger}$, where $\alpha^{\dagger}$ is implicitly defined by

$$
\gamma \frac{\sigma r}{\left(2 \alpha^{\dagger}+r\right)^{2}}=c^{\prime}\left(\alpha^{\dagger}\right)
$$

Advertising is socially excessive, $\alpha^{R *}>\alpha^{P *}>\alpha^{\dagger}$.

\section{Setting 3 (One Firm Has Advantage)}

Consider the setting in which at the beginning of the game $\gamma$ consumers are ignorant and the rest believe brand $A$ is better. As in Setting 2, the primacy and the recency effects give rise to different equilibrium outcomes. Under the primacy effect, the profit functions of the firms are given by

$$
\Pi_{A}^{P}\left(\alpha_{A}, \alpha_{B}\right)=\gamma \frac{\sigma \alpha_{A}}{r\left(\alpha_{A}+\alpha_{B}+r\right)}+(1-\gamma) \frac{\sigma}{r}-\frac{c\left(\alpha_{A}\right)}{r}
$$

and

$$
\Pi_{B}^{P}\left(\alpha_{A}, \alpha_{B}\right)=\gamma \frac{\sigma \alpha_{B}}{r\left(\alpha_{A}+\alpha_{B}+r\right)}-\frac{c\left(\alpha_{B}\right)}{r} .
$$

Under the recency effect,

$$
\Pi_{A}^{R}\left(\alpha_{A}, \alpha_{B}\right)=\gamma \frac{\sigma \alpha_{A}}{r\left(\alpha_{A}+\alpha_{B}+r\right)}+(1-\gamma) \frac{\sigma\left(\alpha_{A}+r\right)}{r\left(\alpha_{A}+\alpha_{B}+r\right)}-\frac{c\left(\alpha_{A}\right)}{r}
$$


and

$$
\Pi_{B}^{R}\left(\alpha_{A}, \alpha_{B}\right)=\frac{\sigma \alpha_{B}}{r\left(\alpha_{A}+\alpha_{B}+r\right)}-\frac{c\left(\alpha_{B}\right)}{r}
$$

Formulas (16) through (19) deserve some discussion. Consider $\gamma$ initially ignorant consumers. The profits that firm $A$ and firm $B$ earn from these consumers are the same under the recency effect as under the primacy (the result obtained in Setting 1). In particular, firm $A$ earns

$$
\gamma \frac{\sigma \alpha_{A}}{r\left(\alpha_{A}+\alpha_{B}+r\right)}
$$

and firm $B$ earns

$$
\gamma \frac{\sigma \alpha_{B}}{r\left(\alpha_{A}+\alpha_{B}+r\right)}
$$

Consider the rest of the consumers that at time $t=0$ prefer brand $A$. These consumers are locked up by firm $A$ under the primacy effect, hence firm $A$ earns

$$
(1-\gamma) \frac{\sigma}{r}
$$

and firm $B$ earns 0 . Under the recency effect firm $A$ loses part of (20) to firm $B$. In particular, firm $A$ earns

$$
(1-\gamma) \frac{\sigma\left(\alpha_{A}+r\right)}{r\left(\alpha_{A}+\alpha_{B}+r\right)}
$$

and firm $B$ earns

$$
(1-\gamma) \frac{\sigma \alpha_{B}}{r\left(\alpha_{A}+\alpha_{B}+r\right)}
$$

Proposition 5 (Equilibrium in Setting 3).

(i) Under the primacy effect $\alpha_{A}^{P *}=\alpha_{B}^{P *}=\alpha^{P *}$, where $\alpha^{P *}$ is implicitly defined by

$$
\gamma \frac{\sigma\left(\alpha^{P *}+r\right)}{\left(2 \alpha^{P *}+r\right)^{2}}=c^{\prime}\left(\alpha^{P *}\right)
$$


(ii) Under the recency effect $\alpha_{A}^{R *}$ and $\alpha_{B}^{R *}$ are implicitly defined by

$$
\left\{\begin{array}{l}
\frac{\sigma\left(\alpha_{B}^{R *}+\gamma r\right)}{\left(\alpha_{A}^{R *}+\alpha_{B}^{R *}+r\right)^{2}}=c^{\prime}\left(\alpha_{A}^{R *}\right), \\
\frac{\sigma\left(\alpha_{A}^{R *}+r\right)}{\left(\alpha_{A}^{R *}+\alpha_{B}^{R *}+r\right)^{2}}=c^{\prime}\left(\alpha_{B}^{R *}\right) .
\end{array}\right.
$$

Also, $\alpha_{A}^{R *}<\alpha_{B}^{R *}<\alpha_{A}^{R *}+(1-\gamma) r$.

(iii) Firm $B$ chooses higher advertising intensity under the recency effect than under the primacy, $\alpha_{B}^{R *}>\alpha^{P *}$.

The first result of Proposition 5 is easily understood. Under the primacy effect $(1-\gamma)$ consumers are locked up by firm $A$. Firm $A$ and firm $B$ are symmetric with respect to $\gamma$ initially ignorant customers, for which they compete. Hence, the firms choose the same advertising intensities in equilibrium, $\alpha_{A}^{P *}=\alpha_{B}^{P *}$.

The result that firm $B$ chooses higher advertising intensity than firm $A$ under the recency effect is also intuitive. At the beginning of the game, firm A's advertising messages affect only $\gamma$ ignorant consumers, as the rest of the consumers already believe brand $A$ is better. Firm $B$ 's messages, on the other hand, affect all consumers - ignorant as well as those who initially preferred brand $A$. Thus, firm $B$ 's incentives to advertise are stronger than firm $A$ 's, especially at the beginning. This results in $\alpha_{B}^{R *}>\alpha_{A}^{R *}$.

Finally, Proposition 5 shows that firm $B$ chooses higher advertising intensity under the recency effect than under the primacy, $\alpha_{B}^{R *}>\alpha_{B}^{P *}=\alpha^{P *}$. Firm $A$ 's equilibrium advertising intensities $\alpha_{A}^{R *}$ and $\alpha_{A}^{P *}=\alpha^{P *}$, however, cannot be ranked, because Firm $A$ 's best reply under the recency effect is non-monotonic in $\alpha_{B}$.

The social welfare function in the current setting coincides with the one obtained in the previous setting,

$$
W\left(\alpha_{A}, \alpha_{B}\right)=\gamma \frac{\sigma\left(\alpha_{A}+\alpha_{B}\right)}{r\left(\alpha_{A}+\alpha_{B}+r\right)}+(1-\gamma) \frac{\sigma}{r}-\frac{1}{r}\left(c\left(\alpha_{A}\right)+c\left(\alpha_{B}\right)\right) .
$$

The next proposition shows that under the primacy effect both firms over-advertise, and, hence, under the recency effect firm $B$ over-advertises ( $\alpha^{\dagger}$ and $\alpha_{A}^{R *}$ cannot be ranked). 
Proposition 6 (Social Optimum in Setting 3). The socially optimal levels of advertising intensities are $\alpha_{A}^{\dagger}=\alpha_{B}^{\dagger}=\alpha^{\dagger}$, where $\alpha^{\dagger}$ is implicitly defined by

$$
\gamma \frac{\sigma r}{\left(2 \alpha^{\dagger}+r\right)^{2}}=c^{\prime}\left(\alpha^{\dagger}\right)
$$

Moreover, $\alpha_{B}^{R *}>\alpha^{P *}>\alpha^{\dagger}$.

\section{Setting 2 versus Setting 3}

The social welfare function is the same in Setting 2 and Setting 3. This makes comparison between the equilibria in the two settings legitimate and, therefore, valuable.

Proposition 7 (Equilibrium in Setting 2 vs. Equilibrium in Setting 3).

(i) Under the primacy effect, the firms' equilibrium advertising intensities in Setting 2 are the same as in Setting 3,

$$
\alpha_{A}^{P *}[\mathrm{~S} 2]=\alpha_{B}^{P *}[\mathrm{~S} 2]=\alpha_{A}^{P *}[\mathrm{~S} 3]=\alpha_{B}^{P *}[\mathrm{~S} 3]
$$

(ii) Under the recency effect, firm B's equilibrium advertising intensity is higher in Setting 3 than in Setting 2,

$$
\alpha_{B}^{R *}[\mathrm{~S} 3]>\alpha_{B}^{R *}[\mathrm{~S} 2]
$$

(The corresponding settings are specified in square brackets.) The first result of Proposition 7 follows directly from Proposition 3(i) and Proposition 5(i). Intuitively, in both settings under the primacy effect the firms compete for $\gamma$ initially ignorant consumers. Whether the rest of the consumers are locked up by both firms, as in Setting 2, or by firm $A$, as in Setting 3, does not change the competition between the firms. Hence, the equilibrium advertising intensities in Setting 2 are the same as in Setting 3.

Next, consider the recency effect. In Setting 3 at the beginning of the game, firm $B$ 's advertising messages affect all consumers, $\gamma$ ignorant and $(1-\gamma)$ that prefer brand $A$. However, 
in Setting 2 firm $B$ 's messages have actual effect only on $\gamma+(1-\gamma) / 2$ consumers - ignorant and those who initially preferred brand $A$. Thus, firm $B$ 's incentives to advertise are stronger in Setting 3. As a result, firm $B$ chooses higher advertising intensity in Setting 3 than in Setting 2 .

\section{Conclusion}

This paper has applied the theories of exposure order effects, developed in psychology literature, to an industrial organization model to explore their role in advertising competition. The equilibrium advertising intensities were characterized separately under the primacy and the recency effects in three different settings. It was shown that in a new market (Setting 1) the advertising equilibrium does not depend on the type of exposure order effect.

However, in a growing market where a number of consumers have already formed their preferences about the two brands (Setting 2), or if one of the firms has more customers than the other (Setting 3), the primacy and the recency effects give rise to different equilibrium outcomes. Under the primacy effect the consumers that prefer a particular brand from the outset will never switch to the other brand. The firms compete for initially ignorant consumers, and, therefore, choose the same advertising intensities across both settings. Under the recency effect, on the other hand, each firm's advertising messages affect ignorant consumers as well as those who prefer the rival brand. In Setting 2 the firms choose higher advertising intensities under the recency effect than under the primacy. In Setting 3 firm $B$ chooses higher advertising intensity than firm $A$. It was also shown that in all three settings the firms over-advertise in equilibrium.

One of the limitations of the present model is that the firms' advertising intensities are fixed throughout the game. A dynamic version of the advertising game, in which the firms choose their advertising intensities continuously, does not have an analytical solution. This is because the differential equations that describe the primacy and the recency effects are not linear in the advertising intensities. ${ }^{6}$ Analyzing the static game under different initial

\footnotetext{
${ }^{6}$ On differential games see, for example, Zaccour (2002) and Erickson (2003).
} 
conditions, however, uncovers some features of the dynamic setup. The number of ignorant consumers, $\gamma$, decreases with the passage of time. Therefore, under the primacy effect the equilibrium advertising intensities gradually converge to zero. Under the recency effect the firms advertise more at the beginning, but their equilibrium advertising intensities will always be bounded away from zero.

\section{References}

[1] Brekke, Kjell Arne, and Mari Rege, 2007, "Advertising as a Distortion of Social Learning," B.E. Journal of Theoretical Economics, vol. 7, iss. 1, article 38.

[2] Brunel, Frédéric F., and Michelle R. Nelson, 2003, "Message Order Effects and Gender Differences in Advertising Persuasion," Journal of Advertising Research, 43, 330-341.

[3] Doob, Joseph L., 1953/1990, Stochastic Processes, 1990 edition, Wiley Classics Library, New York.

[4] Erickson, Gary M., 2003, Dynamic Models of Advertising Competition, 2nd edition, International Series in Quantitative Marketing, Kluwer Academic Publishers, Norwell, MA.

[5] Haugtvedt, Curtis P., and Duane T. Wegener, 1994, "Message order Effects in Persuasion: An Attitude Strength Perspective," Journal of Consumer Research, 21, 205-218.

[6] Krähmer, Daniel, 2004, "Advertising and Consumer Memory," University College London Mimeograph.

[7] Lana, Robert E., 1963, "Interest, Media, and Order Effects in Persuasive Communications," Journal of Psychology, 56, 9-13.

[8] Niedrich, Ronald W., and Scott D. Swain, 2007, "The Effects of Exposure-Order and Market Entry-Information on Brand Preference: A Dual Process Model," Journal of the Academy of Marketing Science, DOI 10.1007/s11747-007-0037-x.

[9] Shapiro, Jesse M., 2006, "A "Memory-Jamming" Theory of Advertising," University of Chicago Mimeograph.

[10] Zaccour, George (ed.), 2002, Optimal Control and Differential Games, Advances in Computational Management Science, vol. 5, Kluwer Academic Publishers, Norwell, MA. 


\section{Appendix}

\section{Derivations of (5) and (6).}

Consider matrix $P$. This matrix has eigenvalues $0,-\left(\alpha_{A}+\alpha_{B}\right)$, 0 . Hence,

$$
P=Q \widetilde{P} Q^{-1},
$$

where

$$
\begin{gathered}
\widetilde{P}=\left(\begin{array}{ccc}
0 & 0 & 0 \\
0 & -\left(\alpha_{A}+\alpha_{B}\right) & 0 \\
0 & 0 & 0
\end{array}\right), \\
Q=\left(\begin{array}{ccc}
0 & 1 & 0 \\
\frac{\alpha_{A}}{\alpha_{A}+\alpha_{B}} & -\frac{\alpha_{A}}{\alpha_{A}+\alpha_{B}} & \frac{1}{\alpha_{A}+\alpha_{B}} \\
\frac{\alpha_{B}}{\alpha_{A}+\alpha_{B}} & -\frac{\alpha_{B}}{\alpha_{A}+\alpha_{B}} & -\frac{1}{\alpha_{A}+\alpha_{B}}
\end{array}\right), \quad Q^{-1}=\left(\begin{array}{ccc}
1 & 1 & 1 \\
1 & 0 & 0 \\
0 & \alpha_{B} & -\alpha_{A}
\end{array}\right) .
\end{gathered}
$$

The columns of $Q$ are right eigenvectors of $P$ and the rows of $Q^{-1}$ are left eigenvectors. The eigenvectors are unique up to a multiplicative constant. The constant in the right eigenvector for eigenvalue 1 is chosen so that it is the invariant probability distribution for $P$,

$$
\left(\begin{array}{c}
0 \\
\frac{\alpha_{A}}{\alpha_{A}+\alpha_{B}} \\
\frac{\alpha_{B}}{\alpha_{A}+\alpha_{B}}
\end{array}\right)
$$

Next, consider matrix $R$. This matrix has eigenvalues $0,-\left(\alpha_{A}+\alpha_{B}\right),-\left(\alpha_{A}+\alpha_{B}\right)$. Hence,

$$
R=Q \widetilde{R} Q^{-1},
$$

where

$$
\widetilde{R}=\left(\begin{array}{ccc}
0 & 0 & 0 \\
0 & -\left(\alpha_{A}+\alpha_{B}\right) & 0 \\
0 & 0 & -\left(\alpha_{A}+\alpha_{B}\right)
\end{array}\right)
$$


$Q$ is the same as above.

Once $P$ and $R$ are diagonalized, it is easy to compute exponentials $e^{t P}$ and $e^{t R}$,

$$
e^{t P}=\sum_{n=0}^{\infty} \frac{Q(t \widetilde{P})^{n} Q^{-1}}{n !}=Q\left(\begin{array}{ccc}
1 & 0 & 0 \\
0 & e^{-\left(\alpha_{A}+\alpha_{B}\right) t} & 0 \\
0 & 0 & 1
\end{array}\right) Q^{-1}=\left(\begin{array}{ccc}
e^{-\left(\alpha_{A}+\alpha_{B}\right) t} & 0 & 0 \\
\frac{\alpha_{A}-\alpha_{A} e^{-\left(\alpha_{A}+\alpha_{B}\right) t}}{\alpha_{A}+\alpha_{B}} & 1 & 0 \\
\frac{\alpha_{B}-\alpha_{B} e^{-\left(\alpha_{A}+\alpha_{B}\right) t}}{\alpha_{A}+\alpha_{B}} & 0 & 1
\end{array}\right)
$$

and

$$
\begin{aligned}
e^{t R} & =\sum_{n=0}^{\infty} \frac{Q(t \widetilde{R})^{n} Q^{-1}}{n !}=Q\left(\begin{array}{ccc}
1 & 0 & 0 \\
0 & e^{-\left(\alpha_{A}+\alpha_{B}\right) t} & 0 \\
0 & 0 & e^{-\left(\alpha_{A}+\alpha_{B}\right) t}
\end{array}\right) Q^{-1} \\
& =\left(\begin{array}{ccc}
e^{-\left(\alpha_{A}+\alpha_{B}\right) t} & 0 & 0 \\
\frac{\alpha_{A}-\alpha_{A} e^{-\left(\alpha_{A}+\alpha_{B}\right) t}}{\alpha_{A}+\alpha_{B}} & \frac{\alpha_{A}+\alpha_{B} e^{-\left(\alpha_{A}+\alpha_{B}\right) t}}{\alpha_{A}+\alpha_{B}} & \frac{\alpha_{A}-\alpha_{A} e^{-\left(\alpha_{A}+\alpha_{B}\right) t}}{\alpha_{A}+\alpha_{B}} \\
\frac{\alpha_{B}-\alpha_{B} e^{-\left(\alpha_{A}+\alpha_{B}\right) t}}{\alpha_{A}+\alpha_{B}} & \frac{\alpha_{B}-\alpha_{B} e^{-\left(\alpha_{A}+\alpha_{B}\right) t}}{\alpha_{A}+\alpha_{B}} & \frac{\alpha_{B}+\alpha_{A} e^{-\left(\alpha_{A}+\alpha_{B}\right) t}}{\alpha_{A}+\alpha_{B}}
\end{array}\right) .
\end{aligned}
$$

\section{Proof of Proposition 1.}

The profit function of firm $i=A, B$ is

$$
\begin{aligned}
\Pi_{i}\left(\alpha_{A}, \alpha_{B}\right) & =\int_{0}^{\infty} e^{-r t} x_{i}(t) \sigma d t-\frac{c\left(\alpha_{i}\right)}{r}=\frac{\sigma \alpha_{i}}{\alpha_{A}+\alpha_{B}} \int_{0}^{\infty}\left(e^{-r t}-e^{-\left(\alpha_{A}+\alpha_{B}+r\right) t}\right) d t-\frac{c\left(\alpha_{i}\right)}{r} \\
& =\frac{\sigma \alpha_{i}}{\alpha_{A}+\alpha_{B}}\left(\frac{1}{r}-\frac{1}{\alpha_{A}+\alpha_{B}+r}\right)-\frac{c\left(\alpha_{i}\right)}{r}=\frac{\sigma \alpha_{A}}{r\left(\alpha_{A}+\alpha_{B}+r\right)}-\frac{c\left(\alpha_{i}\right)}{r} .
\end{aligned}
$$

Firm $i$ 's best reply is implicitly defined by the first order condition for the profit maximization problem,

$$
\frac{\partial}{\partial \alpha_{i}} \Pi_{i}\left(\alpha_{A}, \alpha_{B}\right)=\frac{\sigma\left(\alpha_{j}+r\right)}{r\left(\alpha_{A}+\alpha_{B}+r\right)^{2}}-\frac{c^{\prime}\left(\alpha_{i}\right)}{r}=0,
$$

or

$$
\frac{\sigma\left(\alpha_{j}+r\right)}{\left(\alpha_{A}+\alpha_{B}+r\right)^{2}}=c^{\prime}\left(\alpha_{i}\right)
$$

$i \neq j$. Because

$$
\frac{\partial^{2}}{\partial \alpha_{i}^{2}} \Pi_{i}\left(\alpha_{A}, \alpha_{B}\right)=\frac{-2 \sigma\left(\alpha_{j}+r\right)}{r\left(\alpha_{A}+\alpha_{B}+r\right)^{3}}-\frac{c^{\prime \prime}\left(\alpha_{i}\right)}{r}<0,
$$


the first order condition is sufficient.

Therefore, equilibrium advertising intensities satisfy

$$
\left\{\begin{array}{l}
\frac{\sigma\left(\alpha_{B}^{*}+r\right)}{\left(\alpha_{A}^{*}+\alpha_{B}^{*}+r\right)^{2}}=c^{\prime}\left(\alpha_{A}^{*}\right), \\
\frac{\sigma\left(\alpha_{A}^{*}+r\right)}{\left(\alpha_{A}^{*}+\alpha_{B}^{*}+r\right)^{2}}=c^{\prime}\left(\alpha_{B}^{*}\right) .
\end{array}\right.
$$

The equilibrium is unique and symmetric. Indeed, it follows from above that

$$
\left(\alpha_{A}^{*}+r\right) c^{\prime}\left(\alpha_{A}^{*}\right)=\left(\alpha_{B}^{*}+r\right) c^{\prime}\left(\alpha_{B}^{*}\right)
$$

Because $(\alpha+r) c^{\prime}(\alpha)$ is an increasing function of $\alpha$, it must be the case that $\alpha_{A}^{*}=\alpha_{B}^{*}=\alpha^{*}$, where $\alpha^{*}$ satisfies

$$
\frac{\sigma\left(\alpha^{*}+r\right)}{\left(2 \alpha^{*}+r\right)^{2}}=c^{\prime}\left(\alpha^{*}\right)
$$

Next, observe that the left-hand-side of (21) is decreasing and the right-hand-side is increasing in $\alpha^{*}$. Hence, the solution is unique.

\section{Proof of Proposition 2}

The first order conditions for the social planner's optimization problem are

$$
\left\{\begin{array}{l}
\frac{\sigma r}{\left(\alpha_{A}^{\dagger}+\alpha_{B}^{\dagger}+r\right)^{2}}=c^{\prime}\left(\alpha_{A}^{\dagger}\right) \\
\frac{\sigma r}{\left(\alpha_{A}^{\dagger}+\alpha_{B}^{\dagger}+r\right)^{2}}=c^{\prime}\left(\alpha_{B}^{\dagger}\right)
\end{array}\right.
$$

The matrix of second derivatives

$$
\left(\begin{array}{cc}
-\frac{2 \sigma}{\left(\alpha_{A}+\alpha_{B}+r\right)^{3}}-\frac{c^{\prime \prime}\left(\alpha_{A}\right)}{r} & -\frac{2 \sigma}{\left(\alpha_{A}+\alpha_{B}+r\right)^{3}} \\
-\frac{2 \sigma}{\left(\alpha_{A}+\alpha_{B}+r\right)^{3}} & -\frac{2 \sigma}{\left(\alpha_{A}+\alpha_{B}+r\right)^{3}}-\frac{c^{\prime \prime}\left(\alpha_{B}\right)}{r}
\end{array}\right)
$$


is negative semidefinite, which implies the sufficiency of the first-order conditions. By symmetry, $\alpha_{A}^{\dagger}=\alpha_{B}^{\dagger}=\alpha^{\dagger}$, where $\alpha^{\dagger}$ satisfies

$$
\frac{\sigma r}{\left(2 \alpha^{\dagger}+r\right)^{2}}=c^{\prime}\left(\alpha^{\dagger}\right)
$$

It follows from (21), (22), and the convexity of $c(\alpha)$ that $\alpha^{*}>\alpha^{\dagger}$.

\section{Proof of Proposition 3}

Each part is proven in turn.

(i) Consider the primacy effect. Firm $A$ 's best reply is given by the first order condition

$$
\gamma \frac{\sigma\left(\alpha_{B}+r\right)}{\left(\alpha_{A}+\alpha_{B}+r\right)^{2}}=c^{\prime}\left(\alpha_{A}\right)
$$

firm $B$ 's - by

$$
\gamma \frac{\sigma\left(\alpha_{A}+r\right)}{\left(\alpha_{A}+\alpha_{B}+r\right)^{2}}=c^{\prime}\left(\alpha_{B}\right)
$$

As in the proof of Proposition 1, it can be shown that the first-order conditions are sufficient and that the equilibrium is unique and symmetric, $\alpha_{A}^{P *}=\alpha_{B}^{P *}=\alpha^{P *}$, where $\alpha^{P *}$ satisfies

$$
\gamma \frac{\sigma\left(\alpha^{P *}+r\right)}{\left(2 \alpha^{P *}+r\right)^{2}}=c^{\prime}\left(\alpha^{P *}\right) .
$$

(ii) Consider the recency effect. Firm $A$ 's best reply is given by the first order condition

$$
\gamma \frac{\sigma\left(\alpha_{B}+r\right)}{\left(\alpha_{A}+\alpha_{B}+r\right)^{2}}+\frac{1}{2}(1-\gamma) \frac{\sigma\left(2 \alpha_{B}+r\right)}{\left(\alpha_{A}+\alpha_{B}+r\right)^{2}}=c^{\prime}\left(\alpha_{A}\right)
$$

firm $B$ 's - by

$$
\gamma \frac{\sigma\left(\alpha_{A}+r\right)}{\left(\alpha_{A}+\alpha_{B}+r\right)^{2}}+\frac{1}{2}(1-\gamma) \frac{\sigma\left(2 \alpha_{A}+r\right)}{\left(\alpha_{A}+\alpha_{B}+r\right)^{2}}=c^{\prime}\left(\alpha_{B}\right)
$$

It is straightforward to show that the first-order conditions are sufficient and that the 
equilibrium is unique and symmetric, $\alpha_{A}^{R *}=\alpha_{B}^{R *}=\alpha^{R *}$, where $\alpha^{R *}$ satisfies

$$
\gamma \frac{\sigma\left(\alpha^{R *}+r\right)}{\left(2 \alpha^{R *}+r\right)^{2}}+\frac{1}{2}(1-\gamma) \frac{\sigma}{2 \alpha^{R *}+r}=c^{\prime}\left(\alpha^{R *}\right)
$$

(iii) It follows from (23), (24), and the convexity of $c(\alpha)$ that $\alpha^{R *}>\alpha^{P *}$.

\section{Proof of Proposition 4}

The first order conditions for the social planner's optimization problem are

$$
\left\{\begin{array}{l}
\gamma \frac{\sigma r}{\left(\alpha_{A}^{\dagger}+\alpha_{B}^{\dagger}+r\right)^{2}}=c^{\prime}\left(\alpha_{A}^{\dagger}\right), \\
\gamma \frac{\sigma r}{\left(\alpha_{A}^{\dagger}+\alpha_{B}^{\dagger}+r\right)^{2}}=c^{\prime}\left(\alpha_{B}^{\dagger}\right) .
\end{array}\right.
$$

By symmetry, $\alpha_{A}^{\dagger}=\alpha_{B}^{\dagger}=\alpha^{\dagger}$, where $\alpha^{\dagger}$ satisfies

$$
\gamma \frac{\sigma r}{\left(2 \alpha^{\dagger}+r\right)^{2}}=c^{\prime}\left(\alpha^{\dagger}\right)
$$

It follows from (23), (24), (25), and the convexity of $c(\alpha)$ that $\alpha^{R *}>\alpha^{P *}>\alpha^{\dagger}$.

\section{Proof of Proposition 5}

Each part is proven in turn.

(i) Consider the primacy effect. Firm A's best reply is given by the first order condition

$$
\gamma \frac{\sigma\left(\alpha_{B}+r\right)}{\left(\alpha_{A}+\alpha_{B}+r\right)^{2}}=c^{\prime}\left(\alpha_{A}\right)
$$

firm $B$ 's - by

$$
\gamma \frac{\sigma\left(\alpha_{A}+r\right)}{\left(\alpha_{A}+\alpha_{B}+r\right)^{2}}=c^{\prime}\left(\alpha_{B}\right)
$$

The equilibrium is unique and symmetric, $\alpha_{A}^{P *}=\alpha_{B}^{P *}=\alpha^{P *}$, where $\alpha^{P *}$ satisfies

$$
\gamma \frac{\sigma\left(\alpha^{P *}+r\right)}{\left(2 \alpha^{P *}+r\right)^{2}}=c^{\prime}\left(\alpha^{P *}\right)
$$


(ii) Consider the recency effect. Firm $A$ 's best reply is given by the first order condition

$$
\gamma \frac{\sigma\left(\alpha_{B}+\gamma r\right)}{\left(\alpha_{A}+\alpha_{B}+r\right)^{2}}=c^{\prime}\left(\alpha_{A}\right)
$$

firm $B$ 's - by

$$
\frac{\sigma\left(\alpha_{A}+r\right)}{\left(\alpha_{A}+\alpha_{B}+r\right)^{2}}=c^{\prime}\left(\alpha_{B}\right)
$$

Therefore, $\alpha_{A}^{R *}$ and $\alpha_{B}^{R *}$ satisfy

$$
\left\{\begin{array}{l}
\frac{\sigma\left(\alpha_{B}^{R *}+\gamma r\right)}{\left(\alpha_{A}^{R *}+\alpha_{B}^{R *}+r\right)^{2}}=c^{\prime}\left(\alpha_{A}^{R *}\right), \\
\frac{\sigma\left(\alpha_{A}^{R *}+r\right)}{\left(\alpha_{A}^{R *}+\alpha_{B}^{R *}+r\right)^{2}}=c^{\prime}\left(\alpha_{B}^{R *}\right) .
\end{array}\right.
$$

It is left to show that $\alpha_{A}^{R *}<\alpha_{B}^{R *}<\alpha_{A}^{R *}+(1-\gamma) r$. By contrary, suppose $\alpha_{A}^{R *} \geq \alpha_{B}^{R *}$. Then $c^{\prime}\left(\alpha_{A}^{R *}\right) \geq c^{\prime}\left(\alpha_{B}^{R *}\right)$. It follows from (27) that

$$
\frac{\sigma\left(\alpha_{B}^{R *}+\gamma r\right)}{\left(\alpha_{A}^{R *}+\alpha_{B}^{R *}+r\right)^{2}} \geq \frac{\sigma\left(\alpha_{A}^{R *}+r\right)}{\left(\alpha_{A}^{R *}+\alpha_{B}^{R *}+r\right)^{2}}
$$

or

$$
\alpha_{B}^{R *} \geq \alpha_{A}^{R *}+(1-\gamma) r
$$

This contradicts the supposition. Hence, $\alpha_{A}^{R *}<\alpha_{B}^{R *}$.

Next, suppose $\alpha_{B}^{R *} \geq \alpha_{A}^{R *}+(1-\gamma) r$. Then $c^{\prime}\left(\alpha_{B}^{R *}\right)>c^{\prime}\left(\alpha_{A}^{R *}\right)$. It follows from (27) that

$$
\frac{\sigma\left(\alpha_{A}^{R *}+r\right)}{\left(\alpha_{A}^{R *}+\alpha_{B}^{R *}+r\right)^{2}}>\frac{\sigma\left(\alpha_{B}^{R *}+\gamma r\right)}{\left(\alpha_{A}^{R *}+\alpha_{B}^{R *}+r\right)^{2}},
$$

or

$$
\alpha_{B}^{R *}<\alpha_{A}^{R *}+(1-\gamma) r
$$

This contradicts the supposition. Hence, $\alpha_{B}^{R *}<\alpha_{A}^{R *}+(1-\gamma) r$.

(iii) By contrary, suppose $\alpha^{P *} \geq \alpha_{B}^{R *}$. Then $c^{\prime}\left(\alpha^{P *}\right) \geq c^{\prime}\left(\alpha_{B}^{R *}\right)$. It follows from (26) and the 
second equation in $(27)$ that

$$
\gamma \frac{\sigma\left(\alpha^{P *}+r\right)}{\left(2 \alpha^{P *}+r\right)^{2}} \geq \frac{\sigma\left(\alpha_{A}^{R *}+r\right)}{\left(\alpha_{A}^{R *}+\alpha_{B}^{R *}+r\right)^{2}} .
$$

The right-hand-side of the above inequality is greater than

$$
\frac{\sigma\left(\alpha_{B}^{R *}+r\right)}{\left(2 \alpha_{B}^{R *}+r\right)^{2}}
$$

Indeed,

$$
\frac{\sigma\left(\alpha_{A}^{R *}+r\right)}{\left(\alpha_{A}^{R *}+\alpha_{B}^{R *}+r\right)^{2}}-\frac{\sigma\left(\alpha_{B}^{R *}+r\right)}{\left(2 \alpha_{B}^{R *}+r\right)^{2}}
$$

can be rewritten as

$$
\frac{\sigma\left(\alpha_{B}^{R *}-\alpha_{A}^{R *}\right)\left(\alpha_{B}^{R *}\left(\alpha_{A}^{R *}+r-\alpha_{B}^{R *}\right)+r^{2}+\alpha_{A}^{R *} r\right)}{\left(\alpha_{A}^{R *}+\alpha_{B}^{R *}+r\right)^{2}\left(2 \alpha_{B}^{R *}+r\right)^{2}}>0 .
$$

Therefore, (28) implies

$$
\gamma \frac{\sigma\left(\alpha^{P *}+r\right)}{\left(2 \alpha^{P *}+r\right)^{2}} \geq \frac{\sigma\left(\alpha_{B}^{R *}+r\right)}{\left(2 \alpha_{B}^{R *}+r\right)^{2}} .
$$

Because $(\alpha+r) /(2 \alpha+r)^{2}$ is a decreasing function of $\alpha$, it follows that $\alpha_{B}^{R *}>\alpha^{P *}$. This contradicts the supposition. Hence, $\alpha_{B}^{R *}>\alpha^{P *}$.

\section{Proof of Proposition 6}

The result follows directly from Proposition 3(i), Proposition 4, and Proposition 5(i,iii).

\section{Proof of Proposition 7}

Each part is proven in turn.

(i) The result follows directly from Proposition 3(i) and Proposition 5(i).

(ii) By contrary, suppose $\alpha_{B}^{R *}[S 2] \geq \alpha_{B}^{R *}[S 3]$. Then $c^{\prime}\left(\alpha_{B}^{R *}[S 2]\right) \geq c^{\prime}\left(\alpha_{B}^{R *}[S 3]\right)$. It follows 
from (24) and the second equation in (27) that

$$
\gamma \frac{\sigma\left(\alpha_{B}^{R *}[S 2]+r\right)}{\left(2 \alpha_{B}^{R *}[S 2]+r\right)^{2}}+\frac{1}{2}(1-\gamma) \frac{\sigma}{2 \alpha_{B}^{R *}[S 2]+r} \geq \frac{\sigma\left(\alpha_{A}^{R *}[S 3]+r\right)}{\left(\alpha_{A}^{R *}[S 3]+\alpha_{B}^{R *}[S 3]+r\right)^{2}}
$$

The right-hand-side of the above inequality is greater than

$$
\frac{\sigma\left(\alpha_{B}^{R *}[S 3]+r\right)}{\left(2 \alpha_{B}^{R *}[S 3]+r\right)^{2}}
$$

as was shown in the proof of Proposition 5. Therefore, (29) implies

$$
\gamma \frac{\sigma\left(\alpha_{B}^{R *}[S 2]+r\right)}{\left(2 \alpha_{B}^{R *}[S 2]+r\right)^{2}}+\frac{1}{2}(1-\gamma) \frac{\sigma}{2 \alpha_{B}^{R *}[S 2]+r} \geq \frac{\sigma\left(\alpha_{B}^{R *}[S 3]+r\right)}{\left(2 \alpha_{B}^{R *}[S 3]+r\right)^{2}},
$$

or

$$
\frac{\sigma\left(\alpha_{B}^{R *}[S 2]+r\right)}{\left(2 \alpha_{B}^{R *}[S 2]+r\right)^{2}}-\frac{1}{2}(1-\gamma) \frac{\sigma r}{\left(2 \alpha_{B}^{R *}[S 2]+r\right)^{2}} \geq \frac{\sigma\left(\alpha_{B}^{R *}[S 3]+r\right)}{\left(2 \alpha_{B}^{R *}[S 3]+r\right)^{2}} .
$$

Because $(\alpha+r) /(2 \alpha+r)^{2}$ is a decreasing function of $\alpha$, it follows that $\alpha_{B}^{R *}[S 3]>\alpha_{B}^{R *}[S 2]$. This contradicts the supposition. Hence, $\alpha_{B}^{R *}[S 3]>\alpha_{B}^{R *}[S 2]$. 